\title{
VOR Gain by Head Impulse Video-Oculography Differentiates Acute Vestibular Neuritis from Stroke
}

\author{
*Georgios Mantokoudis, *Ali S. Saber Tehrani, †Amy Wozniak, \\ $\$$ \$Karin Eibenberger, ||Jorge C. Kattah, ||Cynthia I. Guede, *David S. Zee, \\ and $*$ David E. Newman-Toker
}

\begin{abstract}
*Department of Neurology, Johns Hopkins University School of Medicine; †Johns Hopkins Biostatistics Center; and \$Department of Otolaryngology-Head and Neck Surgery, Johns Hopkins University School of Medicine, Baltimore, Maryland, U.S.A.; §University of Applied Sciences Upper Austria, Department of Medical Engineering, Linz, Austria; and /University of Illinois College of Medicine, Peoria, Illinois, U.S.A.
\end{abstract}

\begin{abstract}
Objective: Vestibular neuritis is often mimicked by stroke (pseudoneuritis). Vestibular eye movements help discriminate the two conditions. We report vestibulo-ocular reflex (VOR) gain measures in neuritis and stroke presenting acute vestibular syndrome (AVS).

Methods: Prospective cross-sectional study of AVS (acute continuous vertigo/dizziness lasting $>24 \mathrm{~h}$ ) at two academic centers. We measured horizontal head impulse test (HIT) VOR gains in 26 AVS patients using a video HIT device (ICS Impulse). All patients were assessed within 1 week of symptom onset. Diagnoses were confirmed by clinical examinations, brain magnetic resonance imaging with diffusion-weighted images, and follow-up. Brainstem and cerebellar strokes were classified by vascular territory-posterior inferior cerebellar artery (PICA) or anterior inferior cerebellar artery (AICA).

Results: Diagnoses were vestibular neuritis $(\mathrm{n}=16)$ and posterior fossa stroke (PICA, $\mathrm{n}=7$; AICA, $\mathrm{n}=3$ ). Mean HIT VOR gains (ipsilesional [standard error of the mean], contralesional [standard error of the mean]) were as follows: vestibular neuritis (0.52 [0.04], 0.87 [0.04]); PICA stroke (0.94 [0.04], 0.93
\end{abstract}

[0.04]); AICA stroke (0.84 [0.10], 0.74 [0.10]). VOR gains were asymmetric in neuritis (unilateral vestibulopathy) and symmetric in PICA stroke (bilaterally normal VOR), whereas gains in AICA stroke were heterogeneous (asymmetric, bilaterally low, or normal). In vestibular neuritis, borderline gains ranged from 0.62 to 0.73 . Twenty patients ( 12 neuritis, six PICA strokes, two AICA strokes) had at least five interpretable HIT trials (for both ears), allowing an appropriate classification based on mean VOR gains per ear. Classifying AVS patients with bilateral VOR mean gains of 0.70 or more as suspected strokes yielded a total diagnostic accuracy of $90 \%$, with stroke sensitivity of $88 \%$ and specificity of $92 \%$.

Conclusion: Video HIT VOR gains differ between peripheral and central causes of AVS. PICA strokes were readily separated from neuritis using gain measures, but AICA strokes were at risk of being misclassified based on VOR gain alone. Key Words: Diagnosis-Eye movement measurementsReflex-Stroke-Vertigo-Vestibular neuritis - Vestibulo-ocular.

Otol Neurotol 36:457-465, 2015.
Acute vestibular syndrome (AVS) is a well-described clinical syndrome of new continuous vertigo, nausea/ vomiting, motion intolerance, and gait instability lasting

\footnotetext{
Address correspondence and reprint requests to David E. NewmanToker, M.D., Ph.D., Department of Neurology, The Johns Hopkins University School of Medicine; and The Johns Hopkins Hospital Meyer Bldg 8-154 600 North Wolfe St, Baltimore, MD 21287, U.S.A.; E-mail: toker@jhu.edu

This study was supported by the Swiss National Science Foundation PBBEP2 136573 (G. M.). Drs. Newman-Toker and Kattah have been loaned video-oculography goggles for research studies by GN Otometrics, Inc. (goggles manufacturer). The authors have no financial interest in the company. The company was not involved in the scientific design, conduct, and data analysis or in the decision to publish the manuscript.

Supplemental digital content is available in the text.
}

days to weeks (1). According to a recent systematic review, the most common causes are believed to be vestibular neuritis $(\sim 70 \%)$ and posterior fossa ischemic stroke $(\sim 25 \%)$ (1). Differentiating these two conditions is an important problem facing physicians caring for patients acutely, as in the emergency department (ED). More than 4,000,000 patients with dizziness visit U.S. EDs annually (2). Despite extensive and expensive workups (2), strokes are sometimes missed (3). Such strokes often present isolated AVS (i.e., without focal neurologic signs, found in $<20 \%$ of cases) (4). Thus, central causes often mimic vestibular neuritis closely, leading to use of the term pseudoneuritis to describe neurologic causes of isolated central AVS (5). 
There is now strong evidence that careful bedside oculomotor examination differentiates peripheral vestibular from central causes and even outperforms neuroimaging in the acute phase of the illness $(1,4-7)$. Magnetic resonance imaging (MRI) with diffusion-weighted imaging (DWI) has a sensitivity of approximately $80 \%$ to $85 \%$ if performed in the first 24 to 48 hours $(7,8)$, whereas a noninvasive three-step eye movement test battery with the acronym HINTS (Head Impulse, Nystagmus, Test of Skew) (4) has approximately $99 \%$ sensitivity in diagnosing posterior circulation stroke when performed in the acute setting by subspecialists (7).

The highest sensitivity component of the HINTS battery is the horizontal head impulse test (HIT) of vestibuloocular reflex (VOR) function, with sensitivity $(\sim 91 \%)$ and specificity $(\sim 100 \%)$ for central lesions in AVS - greater than that of MRI-DWI (7). The horizontal HIT consists of a rapid (100-300 degrees per second), passive, lowamplitude (10-20 degrees), unpredictable head movement in the plane of the horizontal canal while the subject fixates on a central target (9). Clinically, a unilaterally abnormal HIT is found in approximately $95 \%$ of patients with vestibular neuritis (1), whereas it is found in less than $10 \%$ of patients with stroke (generally those causing ischemia directly to the labyrinth, vestibular nerve, or vestibular nucleus) (10).

Despite Level $1 \mathrm{~b}$ and strong GRADE evidence supporting the use of the clinical HIT for diagnosis in AVS by experts (1), there are relatively few data quantifying VOR loss in acute patients (11-13). Scleral search coil measurements are rarely available acutely, so, in part, this lack of evidence reflects the evolution of videooculography (VOG) technology, which, until recently, was unreliable for HIT VOR measurement because of goggles slippage (14). Previous studies using newer more reliable VOG-based HIT devices have reported gain values obtained from healthy subjects or from patients with chronic peripheral vestibular deficits (14-19). These results, however, do not necessarily generalize well to VOR measurement for diagnosis of AVS acutely because 1) postacute (partially) recovered VOR deficits in subacute or chronic vestibular neuritis may differ from those with acute vestibular pathology; 2) brisk nystagmus present early on in AVS presentations might influence gain measurements; 3) stroke patients with a negative clinical HIT might have subclinical quantitative VOR deficits; and 4) optimal cut points for distinguishing between neuritis and stroke may differ from optimal cut points for normal versus abnormal in a healthy population.

In this study, we report the distribution of quantitative VOR gains in AVS patients in relation to the underlying cause. The goal of this work is to provide a quantitative framework for developing diagnostic decision tools to differentiate vestibular neuritis and stroke. We hypothesized that VOR gain measures would discriminate neuritis (unilateral VOR deficit) from most strokes (bilateral normal VOR), as seen with nonquantitative, clinical HIT.

\section{MATERIALS AND METHODS}

\section{Study Design, Inclusion, Exclusion Criteria}

We conducted a prospective observational study between August 2011 and December 2012 at two academic medical centers. The design was a cross-sectional comparison of a new test (VOG HIT) with a gold standard final diagnosis (neuroimaging plus clinical follow-up). Through combined active (routine monitoring of ED triage and admission logs) and passive (notified by ED personnel) screening, patients were identified and recruited during daytime hours (Johns Hopkins Hospital, Baltimore, MD, USA) or continuously (OSF St. Francis Medical Center, Peoria, IL, USA). We enrolled adult ED patients with AVS seen less than 7 days since the onset of symptoms and still symptomatic. After ED clinical assessment and a screening interview for eligibility, we included patients with continuous vertigo or dizziness lasting at least 1 hour plus pathologic nystagmus and one or more of the following: 1) nausea or vomiting, 2) head motion intolerance, or 3) new gait or balance disturbance. We excluded patients with known prior vestibular or oculomotor disorder (Ménière's disease, vestibulopathy, oculomotor palsy, etc.), acute drug/alcohol intoxication, or new head trauma based on history and screening examination. As prospectively defined, we also excluded from analysis patients whose symptoms were present at the time of initial assessment but abated in less than 24 hours (not true AVS) and any who lacked MRI-DWI imaging to confirm or exclude stroke (to reduce the risk of diagnostic misclassification). The sample size was determined by availability of patients during the study period. All patients gave written informed consent, and the study was approved by the institutional review boards at both sites.

\section{VOG Devices}

VOR measures were collected at bedside using a portable lightweight VOG device (ICS Impulse, GN Otometrics, Taastrup, Denmark; http://www.icsimpulse.com/) with an integrated digital high-speed camera (250 frames per second) designed for quantitative HIT testing. The ICS device has been validated against dual magnetic scleral search coil results (16), which represent the gold standard for measuring HIT gains. The device software (OTOsuite Vestibular Software Version 1 - 20 Build 310) automatically calculates individual HIT and aggregate (right- and leftsided) mean VOR gains. Inadequate HIT trials are discarded automatically by a proprietary filtering algorithm, and accepted trials de-saccaded before real-time gain calculations. Raw data from accepted impulses can also be exported for offline analysis.

\section{Head Impulse Test}

Patients were seated on a bed or a chair and fixed their gaze on a distant target $(>1.5 \mathrm{~m})$ on the opposite wall or dividing curtain in the ED (Baltimore, MD, USA) or nearby clinic room (Peoria, IL, USA). After calibration, at least 10 inward (i.e., centripetal, lateral-to-center) head impulses in light were performed by the examiner (i.e., passive HITs) directed toward either side in random sequence at an average head velocity of 148 degrees per second (range, 52-348 degrees per second; $\mathrm{SD}, \pm 39$ degrees per second) and an amplitude of approximately 5 to 20 degrees (i.e., target parameters for VOG HIT performance $[14,16])$. Examiners were specially trained postdoctoral research fellows (Baltimore, MD, USA) or a specially trained vestibular clinic nurse (Peoria, IL, USA) who have performed thousands of head impulses. 


\section{Diagnostic Classification}

All AVS patients were classified by two attending-level neuro-otologists and two attending-level neuro-radiologists into one of three diagnostic categories based on clinical assessment and confirmatory investigations: 1) peripheral vestibular, 2) posterior inferior cerebellar artery (PICA) territory stroke, or 3) anterior inferior cerebellar artery (AICA) territory stroke. We prospectively chose to segregate PICA and AICA strokes for analysis because of the high risk of labyrinthine infarction in AICA strokes (10) because the AICA generally gives rise to the internal auditory artery supplying the inner ear, which, in turn, gives rise to the anterior vestibular artery supplying the labyrinth (20). Strokes were all confirmed by MRIDWI within 10 days of symptom onset. Vascular territories were determined radiographically using known vascular distributions for the brainstem and cerebellum (21).

Peripheral vestibular diagnoses were vestibular neuritis (without hearing loss) and labyrinthitis (with hearing loss) and required five criteria: 1) abnormal clinical HIT or bithermal caloric testing $(>25 \%$ asymmetry) $(22,23) ; 2)$ unidirectional spontaneous nystagmus obeying Alexander's law or unilateral gaze-evoked nystagmus with the fast phase directed away from the vestibular deficit (from No. 1); 3) no neurologic signs, including vertical skew deviation; 4) normal or nonspecifically abnormal (e.g., age-related changes) MRI-DWI; and 5) compatible natural history for a minimum follow-up period of 90 days.

\section{Data Analysis}

Although the device software's algorithms reject grossly invalid HITs, some VOG artifacts remain (24). All HITs accepted by the device software were reassessed by two masked, independent, trained human raters offline to ensure that only valid data were included. We presented HITs to raters in random order to prevent interpretation bias. Each HIT was classified as interpretable (no disruptive artifacts or fast eye movements during the VOR) or uninterpretable. Detailed methods and quality standards for assessing artifacts and interpretability are reported elsewhere (24). Uninterpretable impulse data were excluded from all analyses in this article, and we refer to the remaining valid data presented here as filtered VOG HIT results.

For each of the three diagnostic categories (neuritis/labyrinthitis, PICA stroke, AICA stroke), we show histograms of filtered VOG HIT gain results. To account for differences in the number of HITs measured per ear across diagnostic categories, the number of HITs shown in histograms comparing diseases was normalized for each diagnostic category to a uniform per-ear total $(25$, chosen arbitrarily as a typical $n$ ). We report population mean gain values (with standard errors [SEs]) for filtered HIT trials for each side (ipsilesional, contralesional). We used general linear mixed models that accounted for repeated measures in the same patient to calculate confidence intervals (CIs) and $p$ values comparing ipsilesional and contralesional gain values within groups and between groups. We show single-ear VOR gain means (ipsilesional

TABLE 1. Clinical and demographic characteristics of patients presenting AVS

\begin{tabular}{|c|c|c|c|c|c|c|c|c|c|}
\hline \multirow[b]{2}{*}{$\begin{array}{l}\text { Patient } \\
\text { ID }\end{array}$} & \multirow[b]{2}{*}{ Age } & \multirow[b]{2}{*}{ Sex } & \multirow[b]{2}{*}{$\begin{array}{l}\text { Confirmed } \\
\text { diagnosis }\end{array}$} & \multirow[b]{2}{*}{$\begin{array}{l}\text { Lesion } \\
\text { side }\end{array}$} & \multicolumn{3}{|c|}{ HINTS (4) } & \multirow{2}{*}{$\begin{array}{l}\text { Summary } \\
\text { HINTS } \\
\text { result }\end{array}$} & \multirow{2}{*}{$\begin{array}{c}\text { Caloric } \\
\text { asymmetry } \\
(\% / \text { side } \mathrm{CP})\end{array}$} \\
\hline & & & & & $\begin{array}{l}\text { Clinical } \\
\text { HIT }^{a}\end{array}$ & $\begin{array}{l}\text { Direction changing } \\
\text { nystagmus }\end{array}$ & $\begin{array}{l}\text { Vertical } \\
\text { skew }\end{array}$ & & \\
\hline 1 & 66 & $\mathrm{~F}$ & Vestibular neuritis & Left & Abnormal L & No & No & Peripheral & $38 / \mathrm{L}$ \\
\hline 2 & 55 & M & AICA stroke & Right & Abnormal L & Yes & No & Central & NA \\
\hline 3 & 62 & M & PICA stroke & Left & Normal & No & No & Central & NA \\
\hline 4 & 65 & M & Cerebellar peduncle hemorrhage & Left & Normal & Yes & Yes & Central & NA \\
\hline 5 & 31 & M & PICA stroke & Left & Normal & No & No & Central & NA \\
\hline 6 & 72 & M & Vestibular neuritis & Right & Abnormal R & No & No & Peripheral & NA \\
\hline 7 & 61 & M & Vestibular neuritis & Right & Abnormal R & No & No & Peripheral & NA \\
\hline 8 & 62 & $\mathrm{~F}$ & Vestibular neuritis & Right & Abnormal R & No & No & Peripheral & $70 / \mathrm{R}$ \\
\hline 9 & 59 & $\mathrm{~F}$ & Vestibular neuritis & Left & Abnormal L & No & No & Peripheral & $59 / \mathrm{L}$ \\
\hline 10 & 67 & M & Vestibular neuritis & Right & Abnormal R & No & No & Peripheral & NA \\
\hline 11 & 73 & M & Multiple strokes (left AICA stroke) & Bilateral & Abnormal L & No & Yes & Peripheral & NA \\
\hline 12 & 63 & M & PICA stroke & Right & Normal & Yes & No & Central & NA \\
\hline 13 & 46 & M & Vestibular neuritis & Right & Abnormal R & No & No & Peripheral & $51 / \mathrm{R}$ \\
\hline 14 & 83 & M & Vestibular neuritis & Left & Abnormal L & No & No & Peripheral & NA \\
\hline 15 & 76 & M & Labyrinthitis & Left & Abnormal L & No & No & Peripheral & NA \\
\hline 16 & 71 & M & PICA stroke & Right & Normal & No & No & Central & NA \\
\hline 17 & 54 & M & Vestibular neuritis & Right & Abnormal R & No & Yes & Peripheral & NA \\
\hline 18 & 72 & M & Vestibular neuritis & Right & Abnormal R & No & No & Peripheral & NA \\
\hline 19 & 68 & $\mathrm{~F}$ & PICA stroke & Right & Normal & No & No & Central & NA \\
\hline 20 & 43 & M & Vestibular neuritis & Left & Normal $^{b}$ & No & No & Peripheral & $43 / \mathrm{L}$ \\
\hline 21 & 64 & M & PICA stroke & Left & Normal & No & No & Central & NA \\
\hline 22 & 48 & M & Vestibular neuritis & Left & Abnormal L & No & No & Peripheral & $72 / \mathrm{L}$ \\
\hline 23 & 40 & $\mathrm{~F}$ & Vestibular neuritis & Right & Abnormal R & No & No & Peripheral & 9/R \\
\hline 24 & 55 & $\mathrm{~F}$ & Vestibular neuritis & Left & Abnormal L & No & No & Peripheral & NA \\
\hline 25 & 57 & M & Vestibular neuritis & Left & Abnormal L & No & No & Peripheral & NA \\
\hline 26 & 55 & $\mathrm{~F}$ & AICA stroke & Left & Normal & No & No & Central & NA \\
\hline
\end{tabular}

AICA indicates anterior inferior cerebellar artery; AVS, acute vestibular syndrome; CP, canal paresis; F, female; HIT, horizontal head impulse test; $\mathrm{L}$, left; M, male; NA, not available; PICA, posterior inferior cerebellar artery; R, right.

${ }^{a}$ Clinical nonquantitative head impulses were abnormal on the affected side in all but one neuritis case (No. 20) and in two stroke cases with AICAterritory infarctions (No. 2 contralesional HIT; No. 11 ipsilesional HIT).

${ }^{b}$ In this neuritis case, results of caloric testing (abnormal left) and clinical nonquantitative HIT (normal) were dissociated. Quantitative VOG results showed a unilateral deficit on the left side and a borderline normal gain on the fellow side. This patient was unusual in that his symptoms had largely abated by the time testing was obtained (Day 7 after onset). The treating clinician (J. C. K.) believed covert refixation saccades may have been responsible for the falsely normal clinical nonquantitative HIT. 
and contralesional) by disease with CIs (using SEs). Percentile and cumulative distributions, and related analyses are described in the supplemental digital content (http://links.lww.com/MAO/A261). For further analyses relying on individual patient ears (e.g., diagnostic sensitivity and specificity), we excluded ineligible ears with less than five valid filtered HIT trials (11 excluded ears: Patients 1, 9, 17, 21, and 22 and right ear of Patient 2).

Analyses were performed using SAS version 9.2 (SAS Institute Inc, Cary, NC, USA), and a two-sided value of $p<0.05$ was considered statistically significant. For inter-rater agreement, Cohen's $\kappa$ was calculated using SPSS software version 17 (SPSS Inc., Chicago, IL, USA).

\section{RESULTS}

We identified 30 AVS patients and excluded three for lack of confirmatory neuroimaging. In one patient, VOG calibration could not properly be performed. We include for this analysis 26 patients (seven female, 19 male) with a mean age of 60.3 years (SD, $\pm 11.7 \mathrm{yr}$; range, $31-83 \mathrm{yr}$ ), all of whom had either acute peripheral vestibulopathy or stroke. All participants tolerated the HITs and completed the full examination according to the study protocol. Final diagnoses were peripheral vestibular (vestibular neuritis, $\mathrm{n}=15$; labyrinthitis, $\mathrm{n}=1$ [grouped with neuritis here forward]) and posterior fossa stroke (PICA, $n=7$; AICA, $\mathrm{n}=3$ ). Table 1 shows patient demographic and clinical characteristics. We recorded 1,358 unfiltered HITs in 26 patients. Of these, 794 were considered interpretable $(\kappa=$ 0.68 ), and these filtered results form the basis of this analysis. Table 2 shows population mean VOR gains stratified by diagnosis and side. Figures 1 and 2 show population histograms comparing VOR gain values within and between diseases. Figure 3 shows mean single-ear gains for 41 eligible ears after data filtering, stratified by diagnosis. Figure 4 shows patterns of VOR loss in AVS, by disease, for individual patients. Further tabular and graphical analyses of VOR results are available in the supplemental digital content (http://links.lww.com/MAO/A261).

\section{Peripheral Lesions (Vestibular Neuritis/Labyrinthitis) With Unilateral Vestibulopathy}

Peripheral lesions were associated with lower population mean gain values for the affected ear relative to the intact ear (gain, 0.52 [SE, 0.04] versus 0.87 [SE, 0.04]; $p<0.001 ; \mathrm{n}=16)($ Table 2$)$. Although $20 \%$ of individual impulse trials had gain values more than 0.71 (Fig. 1A; eTable 1), single-ear mean gains on the affected side were all 0.73 or less (range, $0.28-0.73 ; \mathrm{n}=12$ ), and the majority ( $\mathrm{n}=8$ of 12) were less than 0.6 (Fig. 3A). All but one contralesional (i.e., presumed normal) ear (No. 20 right ear, gain, 0.62) had a mean gain more than 0.78 . Single-ear mean gains less than 0.62 were only found on the ipsilesional abnormal side. Thus, we conservatively estimate the borderline zone for mean VOR gain in unilateral peripheral vestibulopathy as 0.62 to 0.73 (i.e., range from lowest normal to highest abnormal single-ear mean gain). Just six of 41 eligible ears (i.e., with at least
TABLE 2. Population mean VOR gains in AVS patients by disease and side

\begin{tabular}{|c|c|c|c|c|c|}
\hline Diagnosis & $\begin{array}{c}\text { No. } \\
\text { patients }\end{array}$ & $\begin{array}{l}\text { No. } \\
\text { HITs }\end{array}$ & $\begin{array}{c}\text { Mean VOR } \\
\text { gain }(\mathrm{SE})\end{array}$ & $95 \% \mathrm{CI}$ & $p$ value $^{a}$ \\
\hline Vestibular neuritis & 16 & & & & \\
\hline Ipsilesional & & 195 & $0.52(0.04)$ & $0.44-0.59$ & $<0.001$ \\
\hline Contralesional & & 290 & $0.87(0.04)$ & $0.80-0.94$ & \\
\hline PICA stroke & 7 & & & & \\
\hline Ipsilesional & & 117 & $0.94(0.04)$ & $0.87-1.02$ & .342 \\
\hline Contralesional & & 103 & $0.93(0.04)$ & $0.86-1.00$ & \\
\hline $\begin{array}{l}\text { Ipsilesional/ } \\
\text { contralesional } \\
\text { combined }\end{array}$ & & 220 & $0.94(0.04)$ & $0.84-1.03$ & \\
\hline AICA stroke & 3 & & & & \\
\hline Ipsilesional & & 63 & $0.84(0.10)$ & $0.55-0.94$ & .071 \\
\hline Contralesional & & 26 & $0.74(0.10)$ & $0.64-1.04$ & \\
\hline $\begin{array}{l}\text { Ipsilesional/ } \\
\text { contralesional } \\
\text { combined }\end{array}$ & & 89 & $0.79(0.10)$ & $0.38-1.21$ & \\
\hline Total & & 794 & & & \\
\hline
\end{tabular}

${ }^{a}$ General linear mixed model, comparison between ipsilesional and contralesional VOR gains.

five valid impulses) had mean VOR gains in this borderline range (Fig. 3A).

\section{PICA Strokes With Bilateral Normal VOR}

PICA strokes were associated with tightly clustered consistently normal HIT VOR gains on both sides without VOR asymmetry (Fig. 1C); only 5\% of individual HIT gain values were less than 0.80 (eTable 1). There was no difference between ipsilesional and contralesional population mean gain values $(0.94$ [SE 0.04] versus 0.93 [SE 0.04]; $p=0.342 ; \mathrm{n}=7$ ). Single-ear mean VOR gains $(0.70-1.05$; Fig. $3 \mathrm{~B} ; \mathrm{n}=6)$ were also in the normal range. Figure $2 \mathrm{~A}$ shows the convincing separation in population histograms for VOR gain between ipsilesional vestibular neuritis and ipsilesional VOR gains in PICA stroke patients. These histograms correspond to a large and statistically significant population mean difference versus ipsilesional (abnormal) neuritis gains ( 0.94 versus $0.52 ; p<$ 0.001 ) and no significant difference versus contralesional (normal) neuritis gains ( 0.94 versus $0.87 ; p=0.53$ ).

\section{AICA Strokes With Variable VOR}

AICA strokes, although few in number $(n=3$ patients), were associated with a wide distribution of individual VOR gains (Fig. 1B; eTable 1). The AICA population mean (0.79) was intermediate between the normal (PICA, contralesional neuritis) and abnormal (ipsilesional neuritis) VOR gains (Table 2; eFig. 1). Likewise, the distribution of individual VOR gains overlapped both the neuritis and PICA distributions (Fig. 2B, C). The AICA population mean VOR gain was slightly lower for the contralesional side than the ipsilesional side, although this difference was of borderline statistical significance (contralesional, 0.74 [SE, 0.10] versus ipsilesional, 0.84 [SE, 0.10]; $p=0.07$ ) (Table 2). Importantly, the single-ear mean VOR gains (five eligible ears, $\mathrm{n}=3$ ) indicate 


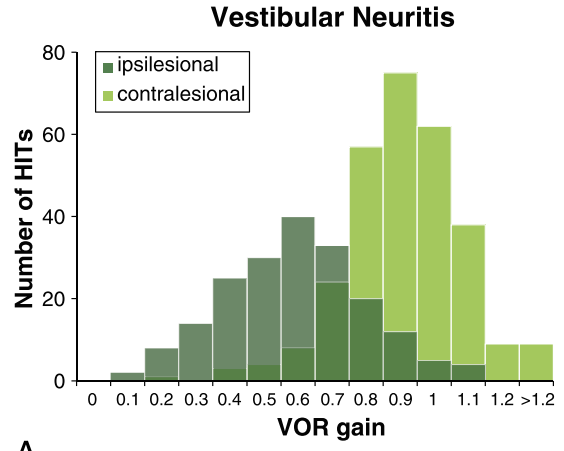

A

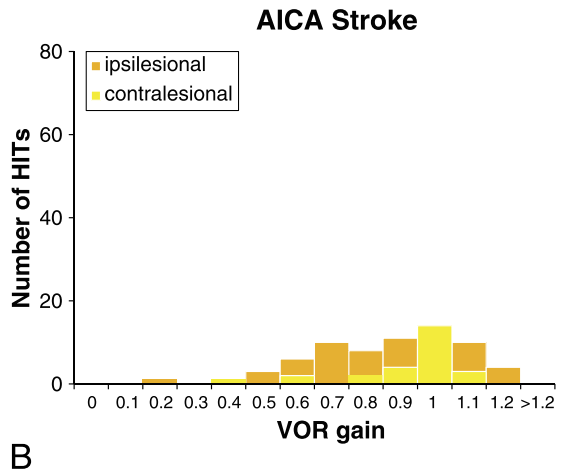

PICA Stroke

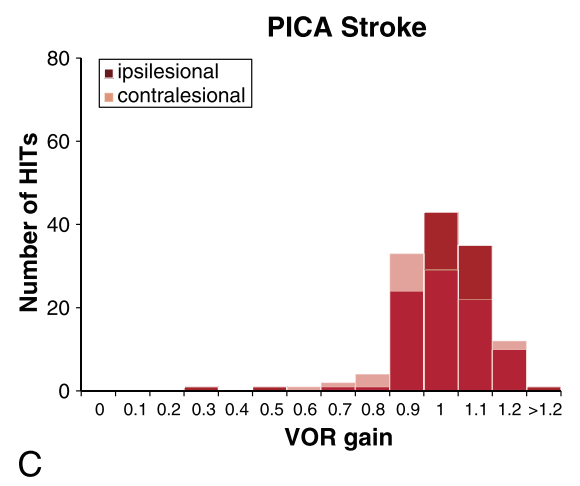

C

FIG. 1. Raw population distributions of VOR gain values for patients with $(A)$ vestibular neuritis, $(B)$ AICA stoke, and $(C)$ PICA stroke. Histograms depict ipsilesional (darker colors) and contralesional (lighter colors) gain values for all valid head impulses in 26 patients with AVS. Data are not normalized to adjust for differences in the number of impulses per ear.

meaningful clinical heterogeneity in AICA vestibular presentations (Fig. 3; eTables 2, 3).

\section{Disease Classification Using VOR Gains}

ROC analyses to develop a gain cutoff for discriminating vestibular neuritis and stroke are shown in Figure 5. Recognizing the asymmetric risk from misclassifying a stroke as opposed to misclassifying vestibular neuritis, the optimal cut point for considering a single-ear mean gain abnormal (i.e., suggestive of vestibular neuritis rather than PICA stroke) was 0.70 . Twenty patients (12 vestibular neuritis, six PICA strokes, two AICA strokes) had at least five interpretable HIT trials (the minimum to calculate single-ear mean VOR gains) for both ears, allowing an appropriate diagnostic classification based on mean VOR gains per ear. Classifying AVS patients with bilateral VOR gains of 0.70 or more as suspected strokes led to a total diagnostic accuracy of $90 \%(\mathrm{n}=18$ of $20 ; 95 \% \mathrm{CI}$, $68 \%-99 \%)$, with a stroke sensitivity of $88 \%(\mathrm{n}=7$ of 8 ; $95 \%$ CI, $47 \%-99 \%)$ and a specificity of $92 \%(n=11$ of 12 ; 95\% CI, 61\%-99\%) (Fig. 5). One AICA stroke case (No. 11) was misclassified as neuritis; this stroke was identifiable by the presence of skew deviation (Table 1). One neuritis case (No. 15) was misclassified as stroke based on a borderline ipsilesional gain of 0.73 , with wide CIs (Fig. 3A); a greater than $20 \%$ right-left VOR gain asymmetry was a strong indicator that the borderline gain value reflected a true VOR deficit (11).

\section{DISCUSSION}

This study reports VOG HIT VOR gain values in peripheral and central vestibular disorders from a cohort of patients with AVS presenting to the ED. We found that VOR deficits were ipsilesional in 15 of 16 vestibular neuritis/labyrinthitis cases, absent in seven PICA stroke cases, and variable in three AICA stroke cases. Using an optimal gain-value cutoff of 0.70 or more bilaterally to predict a central cause of AVS, we were able to correctly classify $90 \%$ of the AVS patients. AICA strokes were at risk of being misclassified based on VOR gain alone but were correctly classified by clinical assessment of other HINTS eye movements (direction changing, gaze-evoked nystagmus, or skew deviation). Our results suggest that
PICA Stroke vs. Vestibular Neuritis

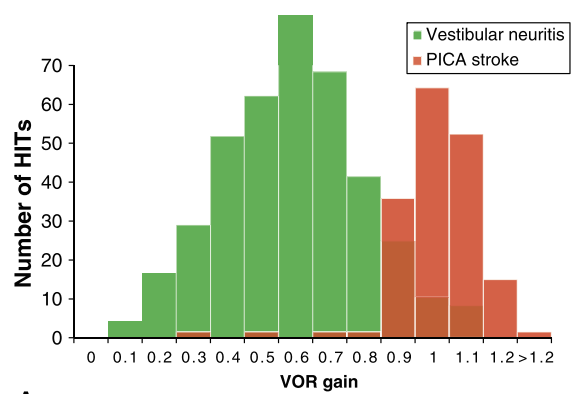

A
AICA Stroke vs. Vestibular Neuritis

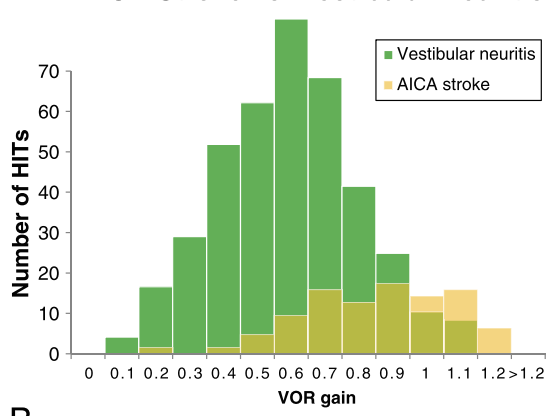

B

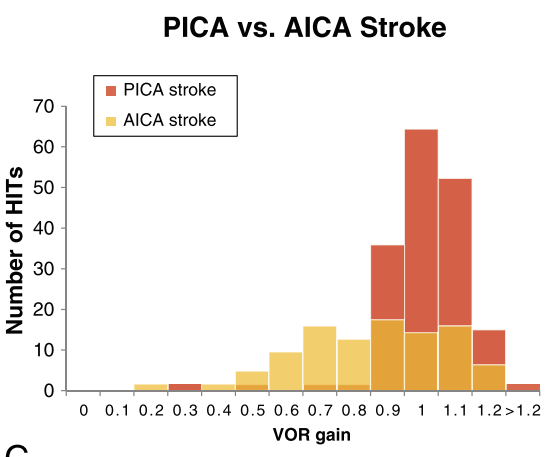

C

FIG. 2. Normalized population distributions of VOR gain values, for each of three primary disease comparisons: $(A)$ vestibular neuritis versus PICA stroke, $(B)$ vestibular neuritis versus AICA stroke, and $(C)$ PICA versus AICA stroke. Histograms depict only ipsilesional gain values in 26 patients with AVS. Shaded colors demonstrate the overlap between two diagnostic groups. Data were normalized to reflect a typical number of HITs per ear (25). 

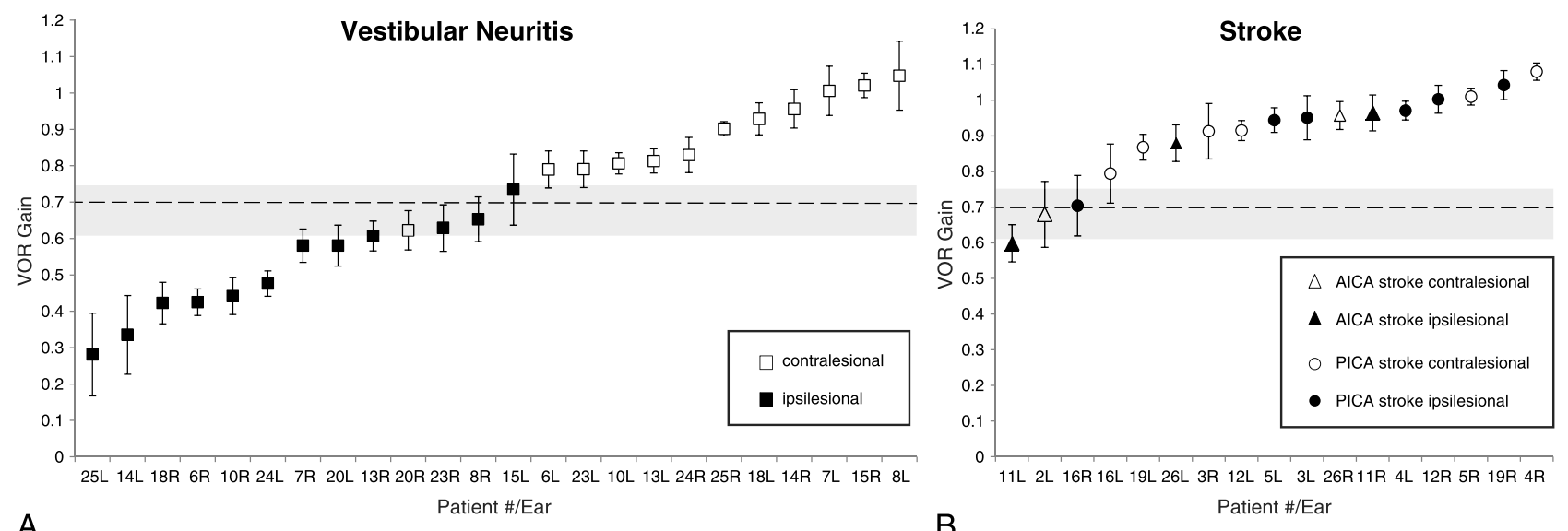

A

FIG. 3. Ipsilesional and contralesional mean VOR gains (with $95 \%$ confidence interval bars) are shown for patients with $(A)$ vestibular neuritis $(n=12)$ and $(B)$ stroke (PICA and AICA together, $n=8)$. Ineligible ears with less than five valid impulses were excluded. The borderline zone (gray shading), defined using a variation in ipsilesional and contralesional mean gains for neuritis patients, indicates gain values that could be either low normal or high abnormal. A dashed line is placed at a gain of 0.70 , the optimized cutoff value for discriminating vestibular neuritis from PICA stroke.

quantitative VOG testing could potentially help physicians diagnose AVS patients in the ED.

\section{Insights for Vestibular Neuritis}

Prior studies of VOR gain in vestibular neuritis have usually not been conducted acutely, and standards for excluding stroke mimics have varied $(16,25,26)$. We studied VOR gains in the early acute period and used a high standard for excluding pseudoneuritis (MRI-DWI plus follow-up). Early assessment is relevant because VOR gain in unilateral vestibular loss depends on the duration after symptoms begin $(27,28)$. VOR gain in vestibular neuritis might change across time because of peripheral recovery or central adaptation (29). Nevertheless,

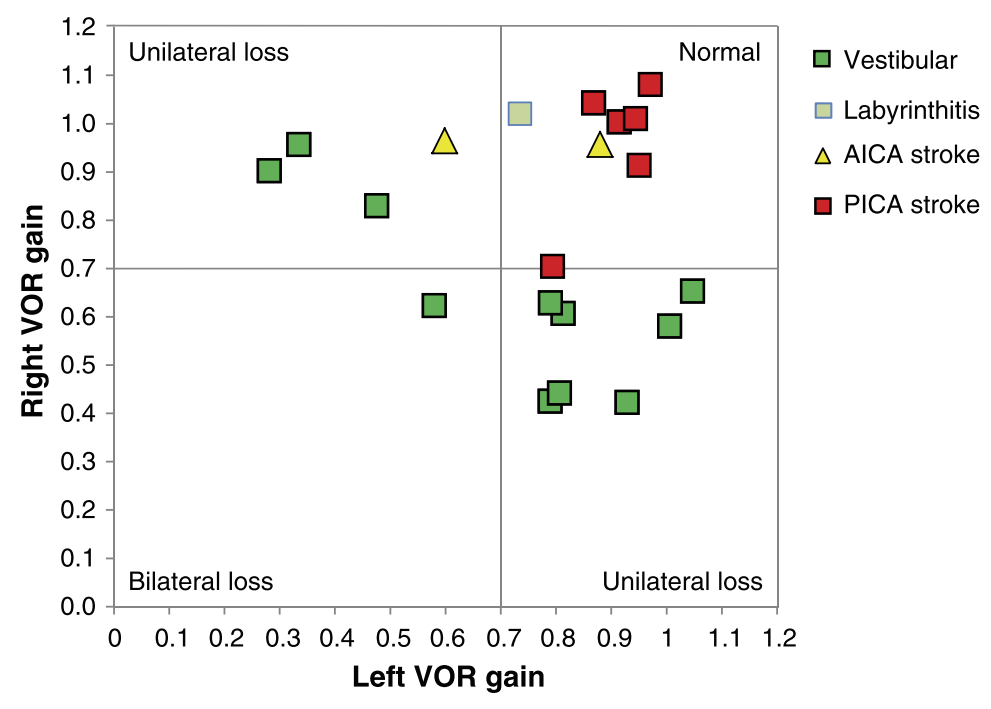

FIG. 4. This patient-level analysis demonstrates the relationship between VOR pattern and underlying diagnosis. Shown is a scatter plot of right-ear mean gain versus left-ear mean gain. Each point on the plot represents a single patient. Only patients with five or more valid horizontal head impulse test (HIT) trials in each ear are shown $(n=20)$. The grid is divided into quadrants by the optimized cutoff of 0.70 for discriminating neuritis and stroke. Strokes are clustered in the upper right quadrant, reflecting bilaterally normal VOR responses. Neuritis cases $(n=12)$ and one pseudoneuritis AICA stroke patient are clustered in either the bottom-right or upper-left quadrants, reflecting unilateral VOR loss on the right or left, respectively. In AVS, ipsilesional and contralesional mean gains must be considered in the context of right-left asymmetry. In fact, there is some evidence to suggest that, in the context of AVS, a relative mean gain asymmetry less than $20 \%$ may predict stroke better than absolute mean gain per se (11). Gain asymmetry can be calculated by normalizing and using the higher gain value as a reference ([higher gain minus lower gain] divided by the higher gain) (11) or by using Jongkees' formula (25), typically used to calculate asymmetry in caloric testing. Because, unlike caloric testing, the input (head velocity) and expected output (eye velocity) is well defined with HIT testing, Jongkees' formula, which uses both ears as a reference for the normalization procedure, is not required (11). 


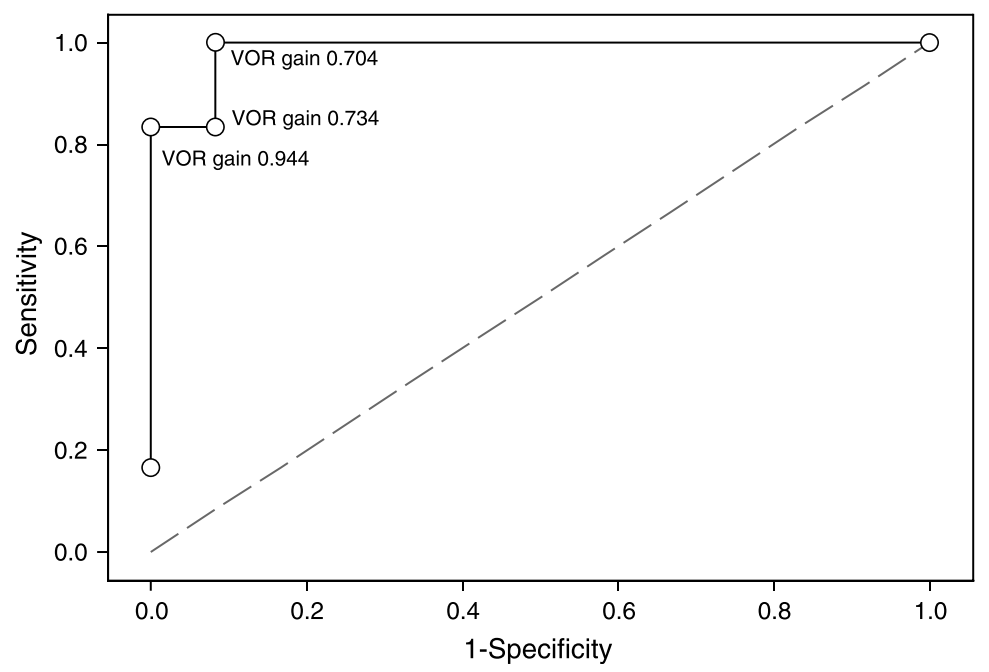

FIG. 5. ROC curve analysis for vestibular neuritis versus PICA stroke. The receiver operating characteristic (ROC) curve illustrates the diagnostic accuracy of quantitative VOG-derived VOR mean gains for discriminating between PICA stroke and vestibular neuritis. We used ipsilesional vestibular neuritis and PICA stroke gains for ROC analysis and to determine an optimal VOR gain cut point for discrimination. We excluded AICA strokes here because gains were heterogeneous. The area under the curve is $0.9861(95 \% \mathrm{Cl}, 0.9476-1.00)$. The dashed diagonal line indicates a hypothetical useless diagnostic test with a likelihood ratio of 1.0 at all diagnostic threshold cut points. Cut point mean VOR gain values corresponding to each stepwise change in sensitivity/specificity are shown here and eTable 4, eFigure 2 , (http://links.Iww.com/MAO/A261). Calculation of overall VOG sensitivity and specificity for stroke detection (including AICA strokes), as presented in the manuscript, was based on using the single-ear mean VOR gain from the lower-measured side (i.e., right or left), applying the optimal cut point derived from the ROC analysis.

our findings confirm that VOR gain failure in the early acute phase (population mean gain, 0.52 ) is similar to that reported previously for patients with subacute or chronic vestibular neuritis. For example, one study of 13 neuritis patients using search coil HITs found that the population mean VOR gain ranged from 0.43 (at 2,000 degrees per second squared head acceleration) to 0.59 (at 750 degrees per second squared) (25).

On the ipsilesional side in neuritis, we found a broad range of gains $(0.18-0.90$ from 5th to 95th percentile) across individual HIT trials and a moderate range of mean gains $(0.28-0.73)$ across ears. Whether these wide ranges reflect frequency-specific gain differences, different degrees of canal paresis, or measurement error/variation is unknown. Regardless, this is important because it makes clear that small numbers of VOG HIT trials (e.g., <5) should probably not be used to define VOR gain in neuritis. Our model-based simulations suggest that 10 or more valid trials should probably be performed for a more robust result, with diminishing marginal gains beyond 25 valid trials per ear (eTable 5).

Search-coil studies have found that the contralesional mean VOR gain (healthy ear) in vestibular neuritis is slightly diminished $(\sim 0.9$ at $750-2,000$ degrees per second squared; $\sim 0.8$ at 4,000 degrees per second squared) (25). According to Ewald's second law, this is explained by a reduction in the inhibitory VOR contribution through commissural vestibular pathways originating from the affected side (30). We found a similar result acutely (population mean gain, 0.87 ), but the difference relative to our population of patients with presumed normal HITs seen in PICA strokes (population mean gain, 0.94) was not statistically significant. The lack of a statistically significant difference may reflect imprecision in the VOG-based measure, the use of a PICA stroke group as (presumed) normal rather than a supernormal (i.e., completely healthy) control reference population, or the relatively small sample size for our comparison, given the known small magnitude of contralesional gain reductions seen in neuritis patients.

\section{Insights for Stroke}

PICA strokes presenting with AVS seem to have quantitatively normal or near-normal VORs by VOG HIT. This finding is consistent with past studies showing normal nonquantitative clinical HIT results in more than $99 \%$ of PICA strokes $(1,7)$. Our population mean VOR gain was 0.94 (SE, 0.04) without side-to-side asymmetry. Prior studies have found similar mean gains using search coils in normal subjects-0.94 (SD, 0.08) (15); 0.98 (SD, $0.06)(25)$. The previously reported mean gain for normal subjects using the ICS Impulse device is lower $(0.81$ [SD, $0.07])(16,31)$, but the corresponding lower $95 \%$ confidence bound on a normal VOG-based VOR gain in that study (0.68) is close to our optimized cutoff derived from the present study $(0.70)$.

Normal HIT VOR results in an AVS population suggest that regions of the lateral medulla and inferior cerebellum supplied by the PICA are generally not required for normal high-frequency VOR function tested by the HIT. Interestingly, however, a prior study found that $22 \%(n=5$ of 23 ) of PICA strokes had abnormal caloric responses despite normal HITs (6). This dissociated loss of caloric function may indicate a functional segregation of 
frequency-specific central pathways. From a clinical diagnostic standpoint, the fact that caloric tests are not well tolerated by patients and not specific for peripheral pathology makes HIT VOR testing the preferred method for predicting central versus peripheral causes of AVS.

By contrast with PICA strokes, AICA strokes causing AVS were more diverse in vestibular pathophysiology, a finding that has been seen previously using nonquantitative clinical HIT testing $(1,4)$. This diversity likely stems from the AICA blood supply to vestibular structures in the brainstem and labyrinth. Ipsilesional abnormal VOR responses in AICA strokes can be caused by infarction of the pontine vestibular nucleus, vestibular nerve root entry zone (fascicle), or labyrinth (10). Contralesional abnormal VOR responses may be caused by flocculus infarction, which can cause an asymmetric bilateral vestibular loss by HIT, worse opposite the affected flocculus (13). If none of these structures is involved, the VOR is usually spared bilaterally.

Some AICA strokes will be difficult to distinguish from vestibular neuritis based on HIT VOR testing alone, and other clinical findings must be sought. A bilateral gaze-evoked component to the nystagmus (mixed vestibular and gaze-holding nystagmus) can distinguish central lesions that may otherwise be exact mimics, including vestibular nucleus infarction (12). A prompt change in the direction of nystagmus after head shaking can also help identify central lesions (32). Although vertical skew deviation (33) has been reported in welldocumented iatrogenic peripheral lesions $(34,35)$, it is present in less than $5 \%$ of cases with confirmed vestibular neuritis. When present, in unselected AVS, skew clearly favors a stroke diagnosis $(1,7)$. Labyrinthine infarctions are usually accompanied by new hearing loss $(36,37)$. Although hearing loss also accompanies labyrinthitis, labyrinthine stroke seems to be more commonly responsible for combined cochleovestibular presentations in unselected ED AVS (7). Facial palsy excludes uncomplicated vestibular neuritis or labyrinthitis and, in the absence of otalgia, a vesicular eruption to suggest herpes zoster oticus, or obvious middle ear pathology, generally indicates a central lesion (4) and typically a lateral pontine infarction $(36,38)$.

\section{Insights for HIT VOR Testing}

Our results suggest that quantitative HIT VOR testing could be used to discriminate central from peripheral causes of AVS in the ED. As a sole method for diagnosis, a search for bilaterally normal VOR gain measures $(\geq 0.70)$ in AVS will have imperfect sensitivity for stroke $(\sim 88 \%)$, but this probably represents an improvement over the current gold standard, MRI-DWI $(\sim 80 \%-85 \%)$, which is more time consuming and substantially more expensive (39). Optimal diagnostic results require consideration of right-left gain asymmetry, especially with borderline mean gain values (0.62-0.73), as illustrated by patient 15 (Fig. 3). Although the VOR gain asymmetry shown in Figure 4 yielded the same sensitivity and specificity as the absolute mean VOR gain, there is some evidence (from our unfiltered mean VOR gain device data) to suggest that, in the context of AVS, a relative mean gain asymmetry of less than $20 \%$ may predict stroke better than absolute mean gain per se (11). More importantly, when combined with other diagnostic eye movements potentially detectable by VOG (gaze-evoked bidirectional nystagmus and skew deviation), stroke sensitivities greater than $99 \%$ might be attainable (7).

\section{LIMITATIONS}

Video systems are limited by their sampling frequency based on camera frame rate (40). Low camera frame rates $(<219$ frames per second according to the ICS device manufacturer's specifications) may predispose to false estimations of eye velocity and acceleration. Although the ICS system has been validated directly against scleral search coils for its accuracy $(14,16,41)$, we did not apply scleral search coils (the gold standard) (42) to confirm VOG results in our patients. Limitations in study design and conduct include nonconsecutive sampling (patients without diagnostic MRI neuroimaging excluded), small sample size, and dropped ears with too few valid HITs to merit inclusion. Although this study's sample size is small, our estimated sensitivity (88\%) and specificity (92\%) are essentially identical to the values previously reported based on pooled data from clinical head impulse testing in 152 stroke patients across several different studies (sensitivity, 0.85 [95\% CI, 0.79-0.91]; specificity, 0.95 [95\% CI, 0.90-1.00]) (1). Nonconsecutive sampling may have biased the mix of patients toward stroke $(38 \%$ versus $25 \%$ reported in the literature) but is unlikely to have affected our conclusions regarding discrimination of neuritis from stroke. Some diseases were not represented (e.g., multiple sclerosis, Ménière's), and clinical findings in these other disorders might differ from those we present. We maximized the validity of our VOG results by filtering invalid impulses not rejected by the ICS device and using highly trained examiners to perform the tests. However, this lowers the immediate generalizability of our findings to device use by nonexperts under normal operating conditions. A future manuscript will address the impact of using unfiltered (i.e., noisy) results.

\section{CONCLUSION}

Quantification of the VOR can be accomplished in an acute care setting with a portable VOG device and specially trained experienced examiners. Vestibular neuritis and stroke can be differentiated based on VOR gain analysis with a high diagnostic accuracy, likely exceeding that of MRI in the first 48 hours after AVS onset. The most accurate diagnostic classification for central pseudoneuritis mimics (especially AICA-territory strokes directly involving primary VOR pathways in the ear or lateral pons) requires assessment of other eye movements and hearing in addition to VOR gain measures. 
Acknowledgments: The authors thank Prof. Mark Shelhamer, Sc.D., for valuable suggestions about VOR gain asymmetries and for the design of Figure 3 and Dr. Gayane Yenokyan for guidance on the statistical methods.

\section{REFERENCES}

1. Tarnutzer AA, Berkowitz AL, Robinson KA, et al. Does my dizzy patient have a stroke? A systematic review of bedside diagnosis in acute vestibular syndrome. CMAJ 2011;183:E571-92.

2. Saber Tehrani AS, Coughlan D, Hsieh YH, et al. Rising annual costs of dizziness presentations to U.S. emergency departments. Acad Emerg Med 2013;20:689-96.

3. Kerber KA, Brown DL, Lisabeth LD, et al. Stroke among patients with dizziness, vertigo, and imbalance in the emergency department: a population-based study. Stroke 2006;37:2484-7.

4. Kattah JC, Talkad AV, Wang DZ, et al. HINTS to diagnose stroke in the acute vestibular syndrome: three-step bedside oculomotor examination more sensitive than early MRI diffusion-weighted imaging. Stroke 2009;40:3504-10.

5. Cnyrim CD, Newman-Toker D, Karch C, et al. Bedside differentiation of vestibular neuritis from central "vestibular pseudoneuritis." J Neurol Neurosurg Psychiatry 2008;79:458-60.

6. Newman-Toker DE, Kattah JC, Alvernia JE, et al. Normal head impulse test differentiates acute cerebellar strokes from vestibular neuritis. Neurology 2008;70:2378-85

7. Newman-Toker DE, Kerber KA, Hsieh YH, et al. HINTS outperforms ABCD2 to screen for stroke in acute continuous vertigo and dizziness. Acad Emerg Med 2013;20:986-96.

8. Oppenheim C, Stanescu R, Dormont D, et al. False-negative diffusion-weighted MR findings in acute ischemic stroke. $A J N R A m$ $J$ Neuroradiol 2000;21:1434-40.

9. Halmagyi GM, Curthoys IS. A clinical sign of canal paresis. Arch Neurol 1988;45:737-9.

10. Kim HA, Lee H. Recent advances in central acute vestibular syndrome of a vascular cause. J Neurol Sci 2012;321:17-22.

11. Newman-Toker DE, Saber Tehrani AS, Mantokoudis G, et al. Quantitative video-oculography to help diagnose stroke in acute vertigo and dizziness: toward an ECG for the eyes. Stroke 2013; 44:1158-61.

12. Kim HJ, Lee SH, Park JH, et al. Isolated vestibular nuclear infarction: report of two cases and review of the literature. J Neurol 2014; 261:121-9.

13. Park HK, Kim JS, Strupp M, et al. Isolated floccular infarction: impaired vestibular responses to horizontal head impulse. J Neurol 2013; 260:1576-82

14. Weber KP, MacDougall HG, Halmagyi GM, et al. Impulsive testing of semicircular-canal function using video-oculography. Ann $N$ Y.Acad Sci 2009;1164:486-91.

15. Halmagyi GM, Aw ST, Cremer PD, et al. Impulsive testing of individual semicircular canal function. Ann N Y Acad Sci 2001;942: 192-200

16. MacDougall HG, Weber KP, McGarvie LA, et al. The video head impulse test: diagnostic accuracy in peripheral vestibulopathy. Neurology 2009;73:1134-41.

17. Bartl K, Lehnen N, Kohlbecher S, et al. Head impulse testing using video-oculography. Ann N Y Acad Sci 2009;1164:331-3.

18. Ulmer E, Bernard-Demanze L, Lacour M. Statistical study of normal canal deficit variation range. Measurement using the Head Impulse Test video system. Eur Ann Otorhinolaryngol Head Neck Dis 2011;128:278-82.

19. Agrawal Y, Schubert MC, Migliaccio AA, et al. Evaluation of quantitative head impulse testing using search coils versus videooculography in older individuals. Otol Neurotol 2014;35:283-8.
20. Oas JG, Baloh RW. Vertigo and the anterior inferior cerebellar artery syndrome. Neurology 1992;42:2274-9.

21. Tatu L, Moulin T, Bogousslavsky J, et al. Arterial territories of human brain: brainstem and cerebellum. Neurology 1996;47: 1125-35.

22. Sills AW, Baloh RW, Honrubia V. Caloric testing 2. results in normal subjects. Ann Otorhinolaryngol Suppl 1977;86:7-23.

23. Fetter M. Caloric testing: background, technique, and interpretation. In: Eggers SDZ, Zee DS, eds. Vertigo and Imbalance: Clinical Neurophysiology of the Vestibular System, Amsterdam, The Netherlands: Elsevier B.V., 2010.

24. Mantokoudis G, Saber Tehrani AS, Kattah JC, et al. Quantifying the vestibulo-ocular reflex with video-oculography: nature and frequency of artifacts. Audiol Neuro-Otol 2014 (in press).

25. Weber KP, Aw ST, Todd MJ, et al. Head impulse test in unilateral vestibular loss: vestibulo-ocular reflex and catch-up saccades. Neurology 2008;70:454-63.

26. Blodow A, Pannasch S, Walther LE. Detection of isolated covert saccades with the video head impulse test in peripheral vestibular disorders. Auris Nasus Larynx 2013;40:348-51.

27. Tian JR, Ishiyama A, Demer JL. Temporal dynamics of semicircular canal and otolith function following acute unilateral vestibular deafferentation in humans. Exp Brain Res 2007;178:529-41.

28. Palla A, Straumann D. Recovery of the high-acceleration vestibuloocular reflex after vestibular neuritis. J Assoc Res Otolaryngol 2004;5:427-35.

29. Mantokoudis G, Schubert MC, Saber Tehrani AS, et al. Early adaptation and compensation of clinical vestibular responses after unilateral vestibular deafferentation surgery. Otol Neurotol 2014; 35:148-54

30. Halmagyi G, Curthoys I, Cremer P, et al. Head impulses after unilateral vestibular deafferentation validate Ewald's second law. $J$ Vestib Res 1990;1:187.

31. Weber KP, Aw ST, Todd MJ, et al. Horizontal head impulse test detects gentamicin vestibulotoxicity. Neurology 2009;72:1417-24.

32. Huh YE, Koo JW, Lee H, et al. Head-shaking aids in the diagnosis of acute audiovestibular loss due to anterior inferior cerebellar artery infarction. Audiol Neuro-Otol 2013;18:114-24.

33. Brodsky MC, Donahue SP, Vaphiades M, et al. Skew deviation revisited. Surv Ophthalmol 2006;51:105-28.

34. Halmagyi GM, Gresty MA, Gibson WP. Ocular tilt reaction with peripheral vestibular lesion. Ann Neurol 1979;6:80-3.

35. Vibert D, Hausler R, Safran AB, et al. Diplopia from skew deviation in unilateral peripheral vestibular lesions. Acta Otolaryngol 1996;116:170-6.

36. Lee H, Kim JS, Chung EJ, et al. Infarction in the territory of anterior inferior cerebellar artery: spectrum of audiovestibular loss. Stroke 2009;40:3745-51.

37. Lee H. Audiovestibular loss in anterior inferior cerebellar artery territory infarction: a window to early detection? J Neurol Sci 2012;313:153-9.

38. Ikegami-Takada T, Izumikawa M, Doi T, et al. AICA syndrome with facial palsy following vertigo and acute sensorineural hearing loss. Auris Nasus Larynx 2012;39:244-8.

39. Newman-Toker DE, McDonald KM, Meltzer DO. How much diagnostic safety can we afford, and how should we decide? A health economics perspective. BMJ Qual Saf 2013;22(Suppl 2):ii11-20.

40. Van der Geest J, Frens M. Recording eye movements with videooculography and scleral search coils: a direct comparison of two methods. J Neurosci Methods 2002;114:185-95.

41. Macdougall HG, McGarvie LA, Halmagyi GM, et al. The Video Head Impulse Test (vHIT) detects vertical semicircular canal dysfunction. PLoS One 2013;8:e61488.

42. Robinson DA. A method of measuring eye movemnent using a scieral search coil in a magnetic field. IEEE Trans Biomed Eng 1963;10:137-45. 